\title{
Desfechos clínicos dos pacientes submetidos à intervenção coronária percutânea com stent bioabsorvível eluidor de everolimus
}

\author{
Clinical outcomes of patients undergoing percutaneous coronary intervention \\ with everolimus-eluting bioabsorbable stent
}

Mariangela Souza Ribeiro ${ }^{1}$, Teresa Cristina Dias Cunha Nascimento ${ }^{2}$, Beatriz Murata Murakami ${ }^{3}$,

Ellen Cristina Bergamasco ${ }^{3}$, Camila Takao Lopes $^{4}$, Eduarda Ribeiro dos Santos 3

\begin{abstract}
RESUMO
Modelo: Estudo descritivo, transversal, retrospectivo. Objetivo: Identificar desfechos clínicos e a prevalência de uso da dupla antiagregação plaquetária (DAP) 30 dias e 12 meses após implante de stent bioabsorvível eluidor de everolimus (BVS). Metodologia e casuística: Foram coletados e analisados de maneira descritiva dados referentes a antecedentes clínicos, procedimento, resultados pós-procedimento e complicações intra-hospitalares, acompanhamento e antiagregação plaquetária 30 dias e um ano após implante de BVS de pacientes submetidos a intervenção coronária percutânea (ICP) com BVS. Resultados: 169 pacientes foram submetidos a 182 a ICP com

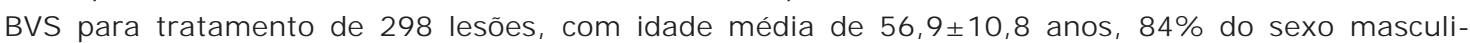
no. Até quatro lesões foram abordadas por ICP, 49\% na artéria descendente anterior, $81,2 \%$ graves (Ellis B2 e C), 99\% em TIMI 3 pós-procedimento. Após o procedimento, o infarto agudo do miocárdio (IAM) sem supradesnivelamento do segmento ST foi a principal complicação intrahospitalar $(11,5 \%)$. Trinta dias após a ICP, não houve complicações relacionadas ao procedimento e 93,4\% estavam em DAP. Após um ano, três pacientes foram submetidos a nova ICP não relacionada ao vaso anteriormente abordado e 80,2\% mantinham DAP. Ressalta-se que não ocorreu re-estenose clinicamente significativa no período de seguimento. Conclusão: A prevalência de uso de DAP foi alta e não houve complicações precoces ou tardias relacionadas à ICP com BVS, confirmando a segurança e efetividade do procedimento na instituição.
\end{abstract}

Palavras-chave: Doença das Coronárias. Intervenção Coronária Percutânea. Stents.

\section{ABSTRACT}

Study design: A descriptive, cross-sectional, retrospective study. Objectives: To identify clinical outcomes and the prevalence of dual antiplatelet therapy (DAPT) 30 days and 12 months after percutaneous coronary intervention $(\mathrm{PCI})$ with an everolimus-eluting bioabsorbable stent (BVS).

1. Graduada em Enfermagem da Faculdade Israelita de Ciências da Saúde Albert Einstein (FICSAE).

2. Coordenadora de Desfecho Clínico do Hospital Sírio Libanês

3. Docente da Graduação em Enfermagem, Medicina e Pós-Graduação da FICSAE

4. Docente da Escola Paulista de Enfermagem, Universidade Federal de São Paulo (EPE/UNIFESP)

Trabalho realizado no Hospital Israelita Albert Einstein
CoRRESPONDÊNCIA: Eduarda Ribeiro dos Santos Av. Professor Francisco Morato, 4293 Vila Sonia,

CEP 05521-200 - São Paulo, SP, Brazil eduarda.santos@einstein.br

Recebido em 26/01/2018

Aprovado em 24/10/2017 
Methods and casuistry: Data regarding clinical history, procedure, post-procedure outcomes and in-hospital complications, 30-day and one-year follow-up and antiplatelet therapy were collected and analyzed of 169 patients submitted to $182 \mathrm{PCls}$ with BVS for treatment of 298 lesions. Results: The mean age was $56.9 \pm 10.8$ years, $84 \%$ male. Up to four lesions were treated with $\mathrm{PCl}, 49 \%$ in the anterior descending artery, $81.2 \%$ severe (Ellis B2 and C), 99\% TIMI 3 postprocedure. After the procedure, non-STEMI was the main in-hospital complication (11.5\%). Thirty days after $\mathrm{PCl}$, there were no procedure-related complications and $93.4 \%$ were on DAPT. After one year, three patients underwent a new $\mathrm{PCl}$ that was not related to the vessel previously treated and $80.2 \%$ maintained DAPT. There were no cases of clinically significant stenosis during the follow-up period. Conclusion: The prevalence of DAPT usage was high and there were no early or late complications related to $\mathrm{PCl}$ with BVS, confirming the safety and effectiveness of the procedure in the institution.

Keywords: Coronary Disease. Percutaneous Coronary Intervention. Stents.

\section{INTRODUÇÃO}

As doenças cardiovasculares (DCV) são um dos principais problemas de saúde pública mundialmente e a causa mais comum de mortalidade nos países desenvolvidos. A doença arterial coronariana (DAC) é responsável por metade dessas mortes. ${ }^{1,2}$

Para o tratamento da DAC, a angioplastia com balão foi um importante marco, porém associada à re-estenose, causada principalmente por remodelamento constritivo. ${ }^{3} \mathrm{Na}$ intervenção coronária percutânea (ICP), os stents metálicos são utilizados como uma resistência estrutural mecânica às limitações da angioplastia com balão, prevenindo o recolhimento elástico precoce do vaso, oclusão aguda e remodelamento constritivo tardio. No entanto, suas complicações, devido à pressão exercida contra a parede do vaso, incluem reestenose tardia secundária à inflamação endotelial crônica, proliferação tecidual focal ou difusa na camada íntima ao longo do stent, com consequente estreitamento do lúmen do vaso, interferência na motricidade e remodelamento vasculares. 3,4,5

Considerando tais limitações, stents farmacológicos substituíram os stents metálicos, liberando agentes antiproliferativos locais nas células do músculo liso, reduzindo, assim, o risco de necessidade de nova revascularização comparado ao risco dos stents metálicos. ${ }^{6,7}$ A cobertura polimérica da superfície dos stents farmacológicos carreia os agentes antiproliferativos e permite sua liberação de maneira controlada, porém, reações ao polímero durável podem desencadear inflamação e trombose tardia do stent. ${ }^{8}$

Assim, novas gerações de stents farmacológicos concentram-se em aumentar a biocompatibilidade dos polímeros e usar polímeros biodegradáveis, que tenham maior capacidade de carrear drogas, de maneira mais direcionada, limitando a proliferação das células musculares lisas, enquanto propiciam a endotelização do lúmen. 3,4,7

O suporte vascular bioabsorvível (BVS) aprovado no Brasil em 2014, Absorb $^{\circledR}$, é constituído por polímeros eluidores da droga everolimus. 0 dispositivo é projetado para restaurar o fluxo de sangue através da abertura do vaso obstruído e sustentar uma força radial suficiente para evitar o recuo elástico do vaso após a dilatação, sendo reabsorvido após dois ou três anos. ${ }^{9,10}$ Para prevenção da trombose de stent, a dupla antiagregação plaquetária (DAP) é indicada por até um ano após o implante de stents farmacológicos ou BVS. ${ }^{11}$

Uma revisão sistemática de 13 estudos em que foram incluídos 3259 pacientes submetidos a implante do Absorb ${ }^{\circledR}$ verificou que a taxa de mortalidade cardíaca entre pacientes com doença arterial coronariana estável foi de 0 a 0,6\%, a taxa de IAM recorrente foi de 0 a $4,5 \%$ e a taxa de trombose do stent foi de 0 a $4, \% .{ }^{12}$

A despeito dos benefícios teóricos dos BVS, revisões sistemáticas de literatura apontam que, comparado ao stent metálico com liberação de everolimus, o BVS pode se associar a maior risco de risco de trombose subaguda, maiores taxas de falência de vaso-alvo e de nova revasculari- 
zação em até três anos após a ICP, com mortalidade similar. As revisões também ressaltam a necessidade de estudos adicionais com maior número de pacientes e maior tempo de acompanhamento. ${ }^{13-16}$

Nesse contexto, é relevante que se conheçam os resultados do implante de BVS especificamente no território brasileiro. Assim, o objetivo deste estudo foi identificar desfechos clínicos e a prevalência do uso de DAP 30 dias e 12 meses após implante de BVS eluidor de everolimus.

\section{MÉTODO}

Tratou-se de um estudo descritivo, transversal, retrospectivo, realizado em uma instituição privada, de extraporte e de alta complexidade, localizada na zona Sul do município de São Paulo. Os dados foram coletados no setor de Cardiologia Intervencionista, que realiza em média 500 ICPS por ano. O setor possui um sistema informatizado de gerenciamento e armazenamento de dados caracterizado como plataforma de imagens e informações, abrangendo todas as especialidades cardiológicas. Este sistema possui informações clínicas basais, do procedimento, até a alta hospitalar, e do acompanhamento clínico após as ICPs.

Os pacientes que realizam angioplastia são acompanhados pelo setor de Pesquisa Clínica da Cardiologia Intervencionista. O contato é realizado via telefone ou e-mail pelas monitoras do setor após 30 dias da alta, após seis meses e anualmente, até completar cinco anos.

Ao longo do acompanhamento, o paciente responde perguntas relativas a sintomas após o procedimento, medicamentos em uso, exames que foram realizados e se foi necessário novo procedimento. Questões relacionadas à ocorrência de acidade vascular encefálico, trombose ou infarto agudo do miocárdio também são realizadas neste contato. Todas as respostas são inseridas no banco de dados.

A população compreendeu os registros dos pacientes que realizaram ICP no período de 24/ 11/2014 (primeiro uso de BVS após aprovação pela Agência Nacional de Vigilância Sanitária) a $30 / 06 / 2016$. Foram incluídos dados de pacientes maiores de 18 anos submetidos à ICP e que utilizaram ao menos um stent BVS no período menci- onado. Foram excluídos registros daqueles que utilizaram, concomitante ao BVS, outro tipo de stent (farmacológico ou metálico) para tratamento das lesões.

Os dados coletados referiram-se a:

I) Dados sociodemográficos: sexo e idade

II) Antecedentes clínicos: apresentação clínica, comorbidades, antecedentes pessoais, antecedentes familiares, medicamentos cardiovasculares de uso contínuo.

III) Dados do procedimento: caráter do procedimento (eletivo, urgência, emergência, salvamento), duração e volume total de contraste da radioscopia, número de lesões abordadas por procedimento, local da lesão, características da lesão tratada, classificação TIMI de fluxo coronariano pré procedimento (TIMI 0 : Sem opacificação após o ponto de obstrução; TIMI 1: Sem opacificação da porção terminal do vaso - Penetração sem perfusão; opacificação completa do vaso, porém em velocidade inferior a vasos adjacentes; TIMI 3: Opacificação total do vaso em velocidade igual dos vasos adjacentes), complexidade coronariana (classificação Ellis), complicações técnicas durante 0 procedimento. ${ }^{17}$

A classificação Ellis seguiu os critérios descritos abaixo. As lesões A e B1 foram agrupadas em "não graves" e as lesões B2 e C foram agrupadas em graves. ${ }^{18}$

A: focais $(<10 \mathrm{~mm})$; concêntricas; acessíveis; em segmento não angulado $(<45 \%)$; de contorno liso; com pouca ou nenhuma calcificação; que não sejam totalmente oclusivas $(<100 \%)$; não ostiais; sem envolvimento de ramos secundários de grande porte; com ausência de trombos.

B1: Uma das seguintes características: comprimento de 10-20mm; excentricidade; moderada tortuosidade do segmento proximal; com segmento moderadamente angulado ( $>45 \circ,<90$ ) ; contorno irregular; moderada ou intensa calcificação; oclusão crônica de $<3$ meses de duração; ostiais; em bifurcação, requerendo técnica de duplo-guia; com trombo presente. 
B2: Duas ou mais das seguintes características: comprimento de $10-20 \mathrm{~mm}$; excentricidade; moderada tortuosidade do segmento proximal; com segmento moderadamente angulado ( $>45 \circ$ ㅇ $<90$ ) ; contorno irregular; moderada ou intensa calcificação; oclusão crônica de $<3$ meses de duração; ostiais; em bifurcação, requerendo técnica de duplo-guia; com trombo presente.

C: difusas ( $>20 \mathrm{~mm}$ de extensão); excessiva tortuosidade do segmento proximal; situadas em segmento extremamente angulado $\left(>90^{\circ}\right)$; oclusões crônicas com $>3$ meses de duração; impossibilidade de se proteger ramo secundário de grande porte emergente da lesão-alvo; pontes de safena degeneradas com lesões friáveis.

IV) Dados intra-hospitalares pós-procedimento: complicações de acordo com os critérios do American College of Cardiology - Cath PCl (morte, AVE, necessidade de nova revascularização, IAM, trombose de stent), classificação TIMI de fluxo coronariano pós procedimento.

V) Acompanhamento clínico: Complicações, conforme julgamento médico individual (IAM, trombose aguda, reparo cirúrgico, estenose/ oclusão arterial, pseudoaneurisma, tamponamento cardíaco, choque cardiogênico) e uso de antiagregantes plaquetários 30 dias e 12 meses após a ICP.

Por meio do teste de Kolmogrov-Smirnov, verificou-se que as variáveis quantitativas tinham distribuição não-paramétrica, exceto pelo volume de contraste. As variáveis qualitativas foram descritas com uso de frequências absolutas e relativas e as variáveis quantitativas foram descritas com uso de medidas de resumo (mediana, mínimo e máximo).

O projeto foi aprovado pelo Comitê de Ética em Pesquisa do Hospital Israelita Albert Einstein, Protocolo $n^{\circ} 1.930 .363$.

\section{RESULTADOS}

No período de estudo, foram realizados 182 ICPs com utilização de ao menos um BVS, trata- mento total de 298 lesões em 169 pacientes, sendo que 13 indivíduos foram submetidos a duas ICPs no período. Para a análise de acompanhamento, foram considerados 106 pacientes, pois foram os que obtiveram acompanhamento de desfecho 30 dias e 12 meses pós-implante de BVS eluidor de everolimus.

Os 169 pacientes submetidos a ICP com BVS tinham idade média de $56,9 \pm 10,8$ anos (34 - 85 anos), a maioria do sexo masculino $(n=142,84 \%)$. Os pacientes apresentavam múltiplas comorbidades (dislipidemia - DLP, hipertensão arterial sistêmica - HAS, diabetes mellitus - DM, IAM). Verificou-se a prevalência de pacientes assintomáticos, sem de dor torácica atípica em sem indicativos de angina instável. A maioria da amostra não apresentou IAM prévio e também não realizou cirurgia de revascularização do miocárdio (CRVM) prévia e utilizava diferentes classes medicamentosas concomitantemente (Tabela 1).

Em relação às características do procedimento, houve ampla variação da duração, até quatro lesões abordadas por procedimento, a maioria das lesões na artéria descendente anterior. Os procedimentos eletivos e de urgência tiveram a mesma prevalência, com apenas 9,9\% de procedimentos de emergência. Quanto à complexidade da DAC, segundo a classificação Ellis, houve predomínio de lesões graves $(n=242$ lesões B2 e C, 81,2\%) (Tabela 2). Destas, 39 $(13,1 \%)$ tratavam-se de re-estenoses. Ressaltase que cada paciente poderia ter apresentado múltiplas lesões com complexidades diferentes.

Em 253 lesões (84,9\%), o fluxo sanguíneo coronário presente no vaso pré-procedimento foi classificado como TIMI 3 e, na avaliação pós-procedimento, o número de lesões com essa classificação aumentou para de 295 (99\%).

Das lesões tratadas, $86,7 \%$ apresentavam estenose pré-procedimento $(60 \%$ a $80 \%$ de oclusão). Em $246(82,6 \%)$ casos optou-se por fazer dilatação com balão antes da liberação do stent. A dilatação com balão após a liberação do stent ocorreu em $239(80,2 \%)$ lesões.

A prevalência de complicações técnicas durante a ICP foi de $2,0 \%(n=6)$. Após o procedimento, IAM sem SST foi a complicação que ocorreu mais frequentemente (Tabela 3 ). 
Tabela 1

Características clínicas dos pacientes submetidos a intervenção coronariana percutânea com stent bioabsorvível eluidor de everolimus $(n=169)$.

\begin{tabular}{|c|c|c|}
\hline \multicolumn{3}{|l|}{ Variável } \\
\hline I dade & mediana & mínimo-máximo \\
\hline & 55 & 34-85 \\
\hline \multirow[t]{2}{*}{ Sexo masculino } & $\mathbf{n}$ & $\%$ \\
\hline & 142 & 84,0 \\
\hline Apresentação clínica & $\mathbf{n}$ & $\%$ \\
\hline Angina instável & 52 & 30,8 \\
\hline Sintomático & 47 & 27,8 \\
\hline Infarto agudo do miocárdio sem SST & 24 & 14,2 \\
\hline Infarto agudo do miocárdio com SST & 20 & 11,8 \\
\hline Dor torácica atípica & 04 & 2,4 \\
\hline Angina estável & 04 & 2,4 \\
\hline Comorbidades & $\mathbf{n}$ & $\%$ \\
\hline Dislipidemia & 118 & 69,8 \\
\hline Hipertensão arterial sistêmica & 94 & 44,4 \\
\hline Angioplastia transluminal por cateter prévia & 40 & 23,7 \\
\hline Tabagismo & 39 & 23,1 \\
\hline Diabetes Mellitus & 36 & 21,3 \\
\hline Antecedentes pessoais & $\mathbf{n}$ & $\%$ \\
\hline Antecedente familiar de doença arterial coronariana & 83 & 50,9 \\
\hline Infarto agudo do miocárdio & 17 & 10,1 \\
\hline Cirurgia de revascularização do miocárdio & 07 & 4,1 \\
\hline Acidente vascular cerebral isquêmico & 02 & 1,2 \\
\hline Acidente isquêmico transitório & 01 & 0,6 \\
\hline Medicamentos de uso contínuo & $\mathbf{n}$ & $\%$ \\
\hline Ácido acetilsalicílico & 165 & 90,7 \\
\hline Estatina & 146 & 80,2 \\
\hline IECA/BRA & 73 & 40,1 \\
\hline Betabloqueador & 71 & 39,0 \\
\hline Clopidogrel & 70 & 38,5 \\
\hline Ticagrelor & 48 & 26,4 \\
\hline Nitratos & 36 & 19,8 \\
\hline Hipoglicemiante oral & 32 & 17,6 \\
\hline Prasugrel & 27 & 14,8 \\
\hline Insulina & 10 & 5,5 \\
\hline
\end{tabular}

IECA: Inibidores de enzima conversora de angiotensina; BRA: Bloqueadores dos receptores da angiotensina; SST: Supradesnivelamento do segmento ST 


\section{Tabela 2}

Características do procedimento de pacientes submetidos a intervenção coronariana percutânea com stent bioabsorvível eluidor de everolimus ( $n=169$ pacientes, 182 procedimentos, 298 lesões).

\begin{tabular}{|c|c|c|}
\hline Variável & Mediana & Mínimo-máximo \\
\hline Duração (min) & 20,3 & $2,1-89,5$ \\
\hline Lesões por procedimento $(n)$ & 1 & $1-4$ \\
\hline Comprimento da lesão (mm) & 24 & $4-96$ \\
\hline \multirow[t]{2}{*}{ Volume de contraste ( $\mathrm{mL})$} & 250 & zero- 550 \\
\hline & $\mathbf{n}$ & $\% *$ \\
\hline \multicolumn{3}{|l|}{ Caráter do procedimento } \\
\hline Eletivo & 82 & 45,1 \\
\hline Urgência & 82 & 45,1 \\
\hline Emergência & 18 & 9,9 \\
\hline \multicolumn{3}{|l|}{ Local da lesão } \\
\hline Artéria descendente anterior & 146 & 49,0 \\
\hline Artéria descendente direita & 80 & 26,8 \\
\hline \multicolumn{3}{|c|}{ Fluxo coronariano pré-procedimento } \\
\hline TIMI 3 & 253 & 84,9 \\
\hline TIMI 0 & 29 & 9,7 \\
\hline TIMI 1 & 9 & 3,0 \\
\hline TIMI 2 & 7 & 2,3 \\
\hline \multicolumn{3}{|c|}{ Fluxo coronariano pós-procedimento } \\
\hline TIMI 3 & 295 & 99,0 \\
\hline TIMI 0 & 3 & 1,0 \\
\hline \multicolumn{3}{|l|}{ Complexidade da DAC (Ellis) } \\
\hline $\mathrm{C}$ & 151 & 50,7 \\
\hline B2 & 91 & 30,5 \\
\hline B 1 & 16 & 5,4 \\
\hline A & 1 & 0,3 \\
\hline
\end{tabular}

*Porcentagem referente ao número de lesões 
Tabela 3.

Complicações intra-hospitalares durante e após intervenção coronariana percutânea com stent bioabsorvível eluidor de everolimus ( $n=169$ pacientes, 182 procedimentos, 298 lesões).

\begin{tabular}{|c|c|c|}
\hline Variável & $\mathrm{n}$ & $\% *$ \\
\hline \multicolumn{3}{|l|}{ Complicações técnicas durante o procedimento } \\
\hline Perfuração de coronária & 5 & 1,7 \\
\hline Dissecção de coronária & 4 & 1,3 \\
\hline Complicações pós-procedimento & $\mathbf{n}$ & $\% * *$ \\
\hline Infarto agudo do miocárdio sem SST & 21 & 11,5 \\
\hline Trombose aguda & 02 & 1,1 \\
\hline Reparo cirúrgico & 02 & 1,1 \\
\hline Infarto Agudo do Miocárdio com SST & 01 & 0,5 \\
\hline Estenose / Oclusão arterial & 01 & 0,5 \\
\hline Pseudoaneurisma & 01 & 0,5 \\
\hline Tamponamento cardíaco com pericardiocentese & 01 & 0,5 \\
\hline Choque cardiogênico refratário seguido de óbito & 01 & 0,5 \\
\hline
\end{tabular}

No acompanhamento de 30 dias após a ICP, não houve complicações relacionadas ao procedimento. Após 12 meses, três $(2,8 \%)$ pacientes foram submetidos a nova ICP em vaso não relacionado à lesão previamente tratada. Ressalta- se que não ocorreu re-estenose clinicamente significativa no período de seguimento.

Trinta dias após a ICP, 99 pacientes (93,4\%) estavam em DAP. Doze meses após o procedimento, 85 pacientes $(80,2 \%)$ mantinham esse padrão.

Tabela 4.

Antiagregantes plaquetários utilizados 30 dias e um ano após a intervenção coronariana percutânea com stent bioabsorvível eluidor de everolimus $(n=106)$.

\begin{tabular}{|c|c|c|c|c|}
\hline \multirow{2}{*}{ Medicamento } & \multicolumn{2}{|c|}{30 dias } & \multicolumn{2}{|c|}{1 ano } \\
\hline & $\mathrm{n}$ & $\%$ & $\mathrm{n}$ & $\%$ \\
\hline AAS e Clopidogrel ou AAS e Ticagrelor & 58 & 54,7 & 46 & 44,7 \\
\hline AAS e Prasugrel & 41 & 38,7 & 39 & 36,7 \\
\hline Nenhum & 3 & 2,8 & 10 & 9,4 \\
\hline Somente AAS & 0 & 0 & 11 & 10,4 \\
\hline Somente Prasugrel & 2 & 1,8 & 0 & 0 \\
\hline Somente Clopidogrel & 1 & 0,94 & 0 & 0 \\
\hline Somente Ticagrelor & 1 & 0,94 & 0 & 0 \\
\hline
\end{tabular}

AAS: Ácido acetilsalicílico 


\section{DISCUSSÃO}

Este estudo verificou ausência de complicações relacionadas à ICP com BVS eluidor de everolimus em curto e longo prazos, além de alta prevalência de adesão à DAP em até 12 meses de seguimento.

Nossos resultados em relação à ausência de IAM prévio e de CRVM prévios são consonantes com estudo realizado para avaliar a qualidade de vida em pacientes com doença multiarterial coronariana que foram submetidos a angioplastia, cirurgia ou tratamento clínico. Este estudo observou, dentre os 483 pacientes avaliados, que somente $66 \%$ referiram no momento da internação a ocorrência de IAM prévio. ${ }^{19} \mathrm{Em}$ outro estudo que avaliou 154.406 angioplastias realizadas entre 2000 e 2005, dos casos estudados entre 20042005, somente $15,98 \%$ apresentavam CRVM prévia. ${ }^{20}$

A associação de re-estenose às lesões graves em nossa amostra representa um desafio para a cardiologia intervencionista, já que se trata de uma resposta vascular à lesão causada pela ICP (remodelamento constritivo). Com o início das angioplastias com o uso de stent farmacológico, a taxa de re-estenose estava presente entre 5 a $10 \%$ dos casos no ano de 2013, um indicador positivo quando comparado ao início do uso dos stents nos anos 80 , quando esta relação estava entre 16 e $44 \% .{ }^{21}$

Os BVS previnem o remodelamento constritivo nos primeiros seis meses após implante, porém permitem o remodelamento expansivo após a perda de sua integridade mecânica. $22,23,24$ Em relação ao remodelamento vascular expansivo, o estudo ABSORB II mostrou que as características basais dos pacientes, como sexo feminino, ICP prévia, maiores níveis de LDL-colesterol e centro necrótico da lesão são determinantes independentes em seguimento de três anos. ${ }^{25}$

A perfuração coronariana como complicação técnica durante o procedimento encontrada associada à abordagem da artéria descendente anterior e lesões do tipo C em nossa amostra também foi verificada em $0,32 \%$ de uma coorte brasileira de 5585 pacientes. A perfuração coronária consiste naquela que apresenta maior potencial de gravidade para o paciente, já que pode ocasionar IAM, tamponamento cardíaco, necessidade de cirurgia de urgência e óbito. ${ }^{26}$

De fato, como complicação intra-hospitalar apresentada pelos pacientes após ICP, prevaleceu o IAM sem SST, em frequência maior que aquela encontrada em estudo realizado entre agosto/ 2011 e outubro de 2013 para acompanhamento tardio de 49 pacientes submetidos à ICP com o uso de BVS, no qual houve registro de $4 \%$ de IAM periprocedimento no período intrahospitalar. ${ }^{10}$

A ausência de desfechos indesejados 30 dias após a ICP em nossa amostra, bem como a necessidade de nova revascularização não relacionada ao vaso anteriormente abordado após 12 meses demonstra eficácia e segurança tardias do BVS e também pode ter relação com a alta prevalência de DAP, em consonância com a recomendação a fim de evitar casos de trombose tardia. ${ }^{27}$

O estudo de coorte ABSORB A acompanhou 30 pacientes tratados com Absorb ${ }^{\circledR}$ durante cinco anos nos Países Baixos e verificou um único episódio de dor torácica com necessidade de nova revascularização, taxa de eventos adversos cardíacos maiores (ECAM) de 3,4\%, ausência de trombose e patência de todos os stents. ${ }^{28} \mathrm{Em}$ seguida, o estudo de coorte ABSORB B mostrou que a bioabsorção ocorreu após três anos em 53 pacientes e associa-se a aumento tardio do lúmen, redução da placa e restauração da motilidade do vaso, com patência de $91 \% .^{24} \mathrm{Na}$ coorte $B$, a taxa de ECAM em cinco anos foi de $11 \%$, sem casos de trombose.

$\mathrm{Na}$ coorte japonesa do ABSORB Japan ( $n=400)$, verificou-se que, em 12 meses, a falência de vaso-alvo foi de $4,2 \%$, com trombose do stent definitiva ou provável de $1,5 \%$ e necessidade de revascularização de lesão-alvo por isquemia de $1,1 \%$. Nesses pacientes, a DAP foi mantida por $81 \%$ dos pacientes aos 12 meses e por $7 \%$ aos cinco anos. ${ }^{29}$

No Brasil, estudo de acompanhamento por até 4,6 anos dos primeiros 49 pacientes submetidos ao implante do Absorb ${ }^{\circledR}$ verificou que o uso do dispositivo foi seguro e eficaz, com $100 \%$ de liberação com sucesso do dispositivo na lesãoalvo e retirada do sistema de entrega do BVS com estenose residual $<50 \%$. Além disso, houve $96 \%$ de casos com sucesso do dispositivo sem a ocorrência de ECAM em até sete dias na internação 
hospitalar. A taxa de ECAM foi de $4 \%$ aos 30 dias, $8,2 \%$ em um ano, e $12,2 \%$ em dois anos e dois casos de trombose até o último seguimento. ${ }^{10}$

As diferentes incidências de ECAM podem ser determinadas pelo tamanho do stent implantado. Uma análise secundária dos dados de 1232 pacientes dos estudos ABSORB B, ABSORB EXTEND e ABSORB II demonstrou que o implante de BVS de grandes dimensões em vasos relativamente pequenos pode se associar a maior taxa de ECAM em até 12 meses, frequentemente devido a IAM. ${ }^{30}$

Os resultados deste estudo são limitados pelo seu caráter unicêntrico. Ademais, a frequência de re-estenose foi investigada exclusivamente por seguimento telefônico e não de maneira ativa, por meio de exames de imagem ou indutores de isquemia.

\section{CONCLUSÃO}

A prevalência de uso da DAP foi alta e não houve complicações precoces ou tardias relacionadas à ICP com BVS, confirmando a segurança e efetividade do procedimento na instituição. Dados sobre os desfechos muito tardios (em até cinco anos) devem ser analisados para confirmar esses achados.

\section{REFERÊNCIAS}

1. World Health Organization. The European health report 2015. Targets and beyond - Reaching new frontiers in evidence. WHO Library Cataloguing in Publication Data, 2015.

2. Benjamin EJ, Blaha MJ, Chiuve SE, Cushman M, Das $S R$, Deo $R$, et al. Heart Disease and Stroke Statistics-2017 Update: A Report From the American Heart Association. Circulation. 2017; 135: e146-e603.

3. Waksman R, Pakala R. Biodegradable and bioabsorbable stents. Curr Pharm Des. 2010; 16: 4041-51.

4. Waksman R. Promise and Challenges of Bioabsorbable Stents. Catheter Cardiovasc Interv 2007;70:40714.

5. Ormiston JA, Serruys PW. Bioabsorbable coronary stents. Circ Cardiovasc Interv. 2009; 2:255-60.

6. Stettler C, Wandel S, Allemann S, et al. Outcomes associated with drug-eluting and bare-metal stents: a collaborative network meta-analysis. Lancet 2007; 370: $937-48$

7. Stefanini GG, Holmes DR Jr. Drug-eluting coronaryartery stents. N Engl J Med. 2013;368:254-65.

8. Lopes Jr AC. Stent bioabsorvível é aprovado para o tratamento da doença arterial coronariana Europa [Internet] Sociedade Brasileira de Cardiologia 2009 [citado 2016 Set 30].
9. Nakazawa G, Finn AV, Joner M, Ladich E, Kutys R, Mont EK, et al. Delayed arterial healing and increased late stent thrombosis at culprit sites after drug-eluting stent placement for acute myocardial infarction patients: an autopsy study. Circulation. 2008; 118: 1138-45.

10. Meneguz-Moreno RA, Costa Junior JR, Moscoso FAB, Staico R, Tanajura LFL, Centemero MP, et al. LongTerm Follow-Up of Patients after Percutaneous Coronary Intervention with Everolimus-Eluting Bioresorbable Vascular Scaffold. Arq Bras Cardiol. 2017; 108: 109-15.

11. Windecker S, Kolh P, Alfonso F, Collet JP, Cremer J, Falk V, et al. 2014 ESC/EACTS Guidelines on myocardial revascularization: The Task Force on Myocardial Revascularization of the European Society of Cardiology (ESC) and the European Association for Cardio-Thoracic Surgery (EACTS) developed with the special contribution of the European Association of Percutaneous Cardiovascular Interventions (EAPCI). Eur Heart J. 2014; 35:2541-619.

12. Keh YS, Yap J, Yeo KK, Koh TH, Eeckhout E. Clinical Outcomes of Bioresorbable Scaffold in Coronary Artery Disease: A Systematic Literature Review. J Interv Cardiol. 2016;29:57-69.

13. Zhang X-L, Zhu L, Wei Z-H, Zhu Q-Q, Qiao Z-Z, Dai $Q$, et al. Comparative Efficacy and Safety of Everolimus-Eluting Bioresorbable Scaffold Versus Everolimus-Eluting Metallic Stents: A Systematic Review and Meta-analysis. Ann Intern Med. 2016; 164:752-63. DOI: 10.7326/M16-0006.

14. Cassese S, Byrne RA, Ndrepepa G, Kufner S, Wiebe J, Repp J, et al. Everolimus-eluting bioresorbable vascular scaffolds versus everolimus-eluting metallic stents: a meta-analysis of randomised controlled trials. Lancet. 2016;387(10018):537-44. doi: 10.1016/S0140-6736(15)00979-4.

15. Ali ZA, Gao R, Kimura T, Onuma Y, Kereiakes DJ, Elis SG, et al. Three-Year Outcomes With the Absorb Bioresorbable Scaffold.Individual-Patient-Data Meta-Analysis From the ABSORB Randomized Trials. Circulation. 2017; 137:464-79

16. Toyota T, Morimoto T, Shiomi H, Yoshikawa Y, Yaku $\mathrm{H}$, Yamashita $\mathrm{Y}$, et al. Very Late Scaffold Thrombosis of Bioresorbable Vascular Scaffold: Systematic Review and a Meta-Analysis. JACC Cardiovasc Interv. 2017; 10(1):27-37. doi: 10.1016/j.jcin.2016.10.027.

17. The TIMI Study Group. The Thrombolysis in Myocardial Infarction (TIMI) trial: phase I findings. N Engl J Med.1984;33:523-30.

18. Ellis SG, Vandormael MG, Cowley MJ, DiSciascio G, Deligonul U, Topol EJ, et al. Coronary morphologic and clinical determinants of procedural outcome with angioplasty for multivessel coronary disease: implications for patient selection. Multivessel Angioplasty Prognosis Study Group. Circulation. 1990; 82:1193-202.

19. Takiuti ME, Hueb W, Hiscock SB, Nogueira CRSR, Girardi P, Fernandes F et al. Qualidade de vida após revascularização cirúrgica do miocárdio, angioplastia ou tratamento clínico. Arq Bras Cardiol. 2007;88: 537-44.

20. Cardoso CO, de Quadros AS, Mattos LA, Gottschall CA, Sarmento-Leite RE, Marin-Neto JA. Perfil de Uso dos Stents Farmacológicos no Brasil: Dados da Central Nacional de Intervenções Cardiovasculares (CENIC). Arq Bras Cardiol. 2007;89:322-6. 
21. Palmerini $T$, Serruys $P$, Kappetein $A P$, Genereux $P$, Riva DD, Reggiani LB, et al. Clinical outcomes with percutaneous coronary revascularization vs coronary artery by-pass grafting surgery in patients with unprotected left main coronary artery disease: a metaanalysis of 6 randomized trials and 4,686 patients. Am Heart J. 2017; 190:54-63.

22. Serruys PW, Onuma $Y$, Ormiston JA, de Bruyne $B$, Regar $E$, Dudek $D$, et al. Evaluation of the second generation of a bioresorbable everolimus drug-eluting vascular scaffold for treatment of de novo coronary artery stenosis: six-month clinical and imaging outcomes. Circulation 2010;122:2301-12.

23. Brugaletta S, Gomez-Lara J, Serruys PW, Farooq V, van Geuns RJ, Thuesen L, et al. Serial in vivo intravascular ultrasound-based echogenicity changes of everolimus-eluting bioresorbable vascular scaffold during the first 12 months after implantation insights from the ABSORB B trial. J Am Coll Cardiol Intv 2011;4:1281-9.

24. Serruys J, Ormiston RJ, van Geuns, de Bruyne B, Regar E, Dudek D, et al. A polylactide bioresorbable scaffold eluting everolimus for treatment of coronary stenosis: 5-year follow-up. J Am Coll Cardiol 2016;67:766-76.

25. Serruys PW, Katagiri $Y$, Sotomi $Y$, Zeng $Y$, Chevalier $B$, van der Schaaf RJ, et al. Arterial Remodeling After Bioresorbable Scaffolds and Metallic Stents. J Am Coll Cardiol. 2017;70(1):60-74. doi: 10.1016/ j.jacc.2017.05.028.

26. Silva WA, Costa RA, Campostrini T, Costa Jr. R, Siqueira DA, Staico R, et al. Incidência, Manejo e Prognóstico de Perfurações Coronárias. Rev Bras Cardiol Invasiva. 2012; 20:295-302.
27. Watti H, Dahal K, Zabher HG, Katikaneni P, Modi K, Abdulbaki A. Comparison of prasugrel and ticagrelor in patients with acute coronary syndrome undergoing percutaneous coronary intervention: a metaanalysis of randomized and non-randomized studies. Int J Cardiol. 2017 249: 66-72.

28. Onuma $Y$, Dudek D, Thuesen L, Webster $M$, Nieman $\mathrm{K}$, Garcia-Garcia HM, et al. Five-year clinical and functional multislice computed tomography angiographic results after coronary implantation of the fully resorbable polymeric everolimus-eluting scaffold in patients with de novo coronary artery disease: the ABSORB cohort A trial. JACC Cardiovasc Interv. 2013;6: 999-1009. doi: 10.1016/j.jcin.2013.05.017.

29. Kimura T, Kozuma K, Tanabe K, Nakamura S, Yamane $M$, Muramatsu $T$, et al. A randomized trial evaluating everolimus-eluting Absorb bioresorbable scaffolds vs. everolimus-eluting metallic stents in patients with coronary artery disease: ABSORB Japan. Eur Heart J. 2015;36:3332-42.

30. Ishibashi $Y$, Nakatani $S$, Sotomi $Y$, Suwannasom $P$, Grundeken MJ, Garcia-Garcia HM, et al. Relation Between Bioresorbable Scaffold Sizing Using QCADmax and Clinical Outcomes at 1 Year in 1,232 $\mathrm{Pa}$ tients From 3 Study Cohorts (ABSORB Cohort B, ABSORB EXTEND, and ABSORB II). JACC CardiovasC Interv. 2015;8:1715-26. doi: 10.1016/ j.jcin.2015.07.026. 